\title{
Analysis of Modified Average Method for One Dimensional Non Linear Burgers Equation with Moving Mesh
}

\author{
Sachin S.Wani ${ }^{1}$, Sarita Thakar $^{2}$ \\ ${ }^{1}$ Department of Applied Mathematics, Pillai's Institute Of Information Technology, Sector 16,Plot No.10,New \\ Panvel,Dist. Raigad,Navi Mumbai(MS) 410206, INDIA, \\ ${ }^{2}$ Department of Mathematics, Shivaji University, Kolhapur (MS) 416004,INDIA
}

\begin{abstract}
A Moving Mesh method is proposed for numerical solution of one dimensional non linear Burgers Equation with homogeneous Dirichlets boundary conditions. Discretization of derivatives is obtained by forward Euler, Backward Euler and central difference formula. We proved that the method is stable in $L_{2}$ norm. Numerical solutions of one dimensional non linear Burgers equation obtained by Modified average method with Moving mesh are bounded. Numerical solutions are given in different domains for different values of $t$ and constant of diffusivity $k$.
\end{abstract}

AMS subject classification:-65M12

Keywords: Burgers equation, Moving mesh, Modified average Method, Discretization, Stability

\section{Introduction}

Moving mesh methods have important applications in a variety of physical and engineering areas such as solid and fluid dynamics, Combustion, heat transfer ,material science etc. Moving mesh method has became an important tool for computing singular or nearly singular problems such as interfaces, shock waves and boundary layers $[\mathbf{1 , 2}]$ and reaction diffusion systems in growing domains [3]. As the domain changes shape, it necessitates the use of a computational mesh that moves and deforms to the new spatial configuration. The numerical investigation of these problems may require extremely fine meshes over a small portion of the physical domain to resolve the large solution variations. Successful implementation of this strategy can increase the accuracy of the numerical approximation and decrease the computational cost.

Burgers equation is a natural first step towards developing methods for control of flows. The one dimensional non linear Burgers equation was first introduced by H. Bateman [4] who found its steady solutions descriptive of certain viscous flows. It was later proposed by J.M.Burger [5] as one of a class of equation describing mathematical model of turbulence. In the context of gas dynamics, it was discussed by E.Hopf [6] and J.D.Cole[7]. N Su (Australia) [8] demonstrates that the Nonlinear Burger's equation can be mapped to the Burnoulli's equation \& Riccati equation respectively. For different convection terms for the analysis of water flows in soils undergoing, surface erasion on hill slopes, Burger's equation is shown to be much easier to use without the need of including the unsaturated hyperbolic conductivity. In 2007 Idris Dag Ali Sahin [9] illustrate how the numerical solution of the Burger's equation is obtained using the method of cubic B-spline collocation \& quadratic B-spline Galerkin over the geometrically graded mesh. In 2005 Ronald E. Mickens[10] gives solutions of several different equation models of Burger's equation for particular cases considered correspond to the diffusion free nonlinear steady -state \& linear steady state situation. In 2008 [11] Alireza , Hashemian , Hossein M Shodja has proposed a new meshless method called gradient reproducing Kernel particle method for the numerical solution of 1-D Burger's equation with various values of viscosity \& different initial and boundary conditions . Discretization is first done in the space via GRKPM \& subsequently the reduced system of nonlinear ordinary differential equation is discretized in time by the Gear's Method. In 2009[12]Sachin wani and Sarita Thakar proved the stability and convergence of Mixed Euler method for one dimensional non linear Burgers equation.

The analysis of numerical methods using moving meshes has received limited attention.. Fezeria (1997) [13] analysed a moving mesh implicit Euler method that uses central finite differences to descretize the spatial derivatives of model linear convection reaction diffusion problems in 1-d using an energy type analysis . It was shown that the method was only conditionally stable depending on the temporal and spatial smoothness of the moving mesh. Ferrerra then went to show that the mesh would be sufficiently smooth if it was obtained by the equidistribution of a smooth monitor function. The result is rather surprising given that the method is fully implicit \& is unconditionally stable when the mesh is stationary.

An important issue related to the use of moving mesh techniques to solve conservation laws is so called Geometric conservation Laws (GCL) which was first introduced by Thomas \& Lambard (1979) [14]. In 1990 [15] R. M. Fuzzeland, J. G. Verwer gave their moving mesh techniques which is due to the moving finite 
difference method proposed by Dorfi \& Drury [16] . The spatial discretization is done by a second order nonlinear Galerkin based method which is studied extensively in Skeel and Berzins[17].Some practical aspects of formulations and solutions of moving mesh PDE's are reviewed in Huang[18]. One of the successful applications of the moving mesh PDE's approach is given in [19] to solve one dimensional PDE's with blow up solution. In 2002 [20] Dupont and co-workers \& in 2003 Lie etal [21] were analysed certain moving mesh finite element methods for a model advection diffusion equation .In 2004 [22]Zhijum Tan,Zhengru Zhang, Yunging Huang Tang solved physical PDE on a fixed mesh and the effect of the mesh movement is achieved through the grid restructuring .However the stability restriction on a fixed mesh will be governed by the smallest mesh size and therefore a local time staping technique seems necessary for this type of moving mesh methods .In 2007 [23] J.A.Mackenzie \& W.R Mekwi analysis the stability and convergence of a finite difference discretization of a model parabolic PDE in 1D using moving mesh In this spatial discretization is achieved using a second order central finite difference scheme and showed that Euler scheme is unconditionally stable in mesh dependent $L_{2}$ norm, independent of the mesh movement .They also derived an adaptive $\theta$ method that is shown to be unconditionally stable and second order accurate.

In this paper we construct a Modified average method for one dimensional nonlinear Burgers equation with Homogeneous Dirichlets boundary conditions with Moving Mesh. In this discretization is achieved by approximating linear term by Backward difference and nonlinear term by Forward difference formula. We proved that the method combines Mixed Euler and forward Euler schemes in such a way that the resulting scheme is stable in $L_{2}$ norm. The boundedness of error is achieved and it is shown that the solutions are bounded.

The paper is arranged as follows. In section 2 we present the model problem and its reformulation with respect to moving coordinate frame. In section 3, the moving mesh discretization is given. In section 4 stability of Modified average method using moving mesh is analysed. Finally in section 5 we present numerical solution of Modified average method in different domains for different values of $t$ and constant of diffusivity $k$.

\section{Model Problem}

Let $\mathrm{T}>0$ and for each $t \in[0, T], \Omega t$ be a domain in R. We shall use the notation $Q_{T}=\left\{(x, t) \in R^{2}: x \in \Omega t, t \in(0, T)\right\}$

We consider non linear one dimensional Burger's Equation

$$
\begin{gathered}
u_{t}+u u_{x}=k u_{x x} \\
\text { i.e } u_{t}+\frac{\partial}{\partial x}\left(\frac{1}{2} u^{2}-k \frac{\partial u}{\partial x}\right)=0 \\
(x, t) \in Q_{T} \\
u=u_{0}, x \in \Omega_{0}, t=0 \\
u=0, x \in \partial \Omega, t>0
\end{gathered}
$$

Where $\mathrm{k}$ is a constant of diffusivity.

We will assume that $\Omega_{0}$ is bounded and $u_{0}$ is sufficiently smooth Let $A_{t}$ be a family of mappings, which at each $t \in[0, T]$ maps the points $\xi$ of a reference or computational domain $\Omega_{c}$ in to the points of domain $\Omega_{t}$ at time t. Then for each $t \in[0, T], A_{t}: \Omega_{c} \rightarrow \Omega_{t}, x(\xi, t)=A_{t}(\xi)$.

We assume that $A_{t}$ is a bijective and $\Omega_{t}=A_{t}\left(\Omega_{c}\right)$ is a bounded for a function $g: Q_{T} \rightarrow R$ defined on the physical domain, The time derivative in the reference domain is

$$
\left.\dot{g} \equiv \frac{\partial g}{\partial t}\right|_{\xi}: Q_{T} \rightarrow R
$$

If $u: Q_{T} \rightarrow R$ is regular enough ,then by chain rule $\dot{u}=\left.\frac{\partial u}{\partial t}\right|_{x}+\left.\dot{x} \frac{\partial u}{\partial x}\right|_{x}$ The governing equation (2.1) in the computational domain therefore takes the form

$$
\begin{gathered}
\dot{u}-k u_{x x}-\dot{x} u_{x}+\frac{1}{2} \frac{\partial}{\partial x}\left(u^{2}\right) \\
u=u_{0}, x \in \Omega_{0}, t=0
\end{gathered}
$$




$$
u=0, x \in \partial \Omega_{t}, t>0
$$

Now we will write the conservative form of (2.2)

$$
\left(x_{\xi} u\right)-\left(\dot{x} u-\frac{1}{2} u^{2}+k \frac{u_{\xi}}{x_{\xi}}\right) \xi=0
$$

\section{Moving Mesh Discretizations}

We consider the semi-discretization of (2.3) achieved using second order central finite -difference approximations of the spatial derivatives of $\mathrm{u}$ and a discretization of the mesh velocity and meshmetric terms. We will assume that the domain $\Omega_{t}=\left[x_{l}(t), x_{r}(t)\right]$ is covered by a nonuniform mesh of $\mathrm{N}$ cells with $x_{l}(t)=x_{0}(t)<x_{1}(t)<------<x_{N-1}(t)<x_{N}(t)=x_{r}(t)$

The nonuniform moving mesh in physical space is assumed to be the image of a fixed uniform mesh covering the computational domain $\Omega_{0}=[0,1]$.via the mapping $x(\xi, t)$,so that $x_{j}(t)=x\left(\xi_{j}, t\right)=x(j / n, t), \mathrm{j}=0,1,--$ $---\mathrm{N}$.

The measure of each physical cell will be denoted by

$h_{j}(t)=x_{j}(t)-x_{j-1}(t) . \mathrm{j}=1,2-----\mathrm{N}$ and the midpoints of the cell are defined as $x_{j-1 / 2}(t)=$ $\frac{1}{2}\left(x_{j}(t)+x_{j-1}(t)\right) \cdot \mathrm{j}=1,2-----\mathrm{N}$

The location of the physical mesh points at time level $t=t_{n}$ and $t=t_{n+1}$ is well defined given the mapping $x(\xi, t)$.To obtain the numerical approximation of (2.3) we require an approximation $x^{h}(\xi, t)$ of $x(\xi, t)$.We will assume that $x^{h}(\xi, t)$ is piecewise linear in space and time and hence

$$
\begin{aligned}
& x^{h}(\xi, t)=x_{j-1 / 2}(t)+\left(\xi-\xi_{j-1 / 2}\right)\left(x_{j+1 / 2}(t)+x_{j-1 / 2}(t)\right) N \text { for } \xi_{j-1 / 2} \leq \xi \leq \xi_{j+1 / 2} \text { and } \\
& x_{j-1 / 2}(t)=x_{j-1 / 2}\left(t_{n}\right)+\left(t-t_{n}\right)\left(\frac{x_{j-1 / 2}\left(t_{n+1}\right)-x_{j-1 / 2}\left(t_{n}\right)}{t_{n+1}-t_{n}}\right), t_{n} \leq t \leq t_{n+1}
\end{aligned}
$$

Assuming this form for the mapping we have

And

$$
x_{\xi}^{h}\left(t_{n}\right)=\left(\frac{x_{j+1 / 2}\left(t_{n}\right)-x_{j-1 / 2}\left(t_{n}\right)}{\Delta \xi}\right)=\frac{1}{\Delta \xi}\left(\frac{h_{j+1}^{n}+h_{j}^{n}}{2}\right) \xi_{j-1 / 2} \leq \xi \leq \xi_{j+1 / 2}
$$

$$
\dot{x}_{j-1 / 2}^{h}(t)=\left(\frac{x_{j-1 / 2}\left(t_{n+1}\right)-x_{j-1 / 2}\left(t_{n}\right)}{t_{n+1}-t_{n}}\right), t_{n} \leq t \leq t_{n+1}
$$

With these definitions, it is easy to see that $x^{h}(\xi, t)$ satisfies the DGCL, which states that

$$
\left(x_{\xi}^{h}\right)_{j}^{n+1}=\left(x_{\xi}^{h}\right)_{j}^{n}+\frac{\left(t_{n+1}-t_{n}\right)}{\Delta \xi}\left(\dot{x}_{j+1 / 2}^{h}-\dot{x}_{j-1 / 2}^{h}\right)
$$

To define the semidicretization of (2.3).We will use the notation $u_{j}^{n}$ to denote the approximation of $u\left(x_{j}^{n}, t_{n}\right)$ and $\mathbf{u}^{n}=\left(u_{0}^{n}, u_{1}^{n}---u_{N-1}^{n}, u_{N}^{n}\right)^{T}$.We will use the forward and backward divided differences $\left(D_{+} \mathbf{u}\right)_{j}=\frac{u_{j+1}-u_{j}}{h_{j+1}},\left(D_{-} \mathbf{u}\right)_{j}=\frac{u_{j}-u_{j-1}}{h_{j}}$ average operator $(\delta \mathbf{u})_{j+1 / 2}=\frac{1}{2}\left(u_{j}+u_{j+1}\right)$ and the central divided difference $\mu \mathbf{u}_{j}^{n}=\frac{u_{j+1}^{n}-u_{j-1}^{n}}{h_{j+1}+h_{j}}$

Using the notations (3.1) and (3.2), the semidiscretization of (2.3) takes the form 
$\left(x_{\xi}^{h} u\right)_{j}=\frac{1}{\Delta \xi}\left[\left(k\left(D_{+}-D_{-}\right) \mathbf{u}\right)_{j}+\dot{x}_{j+1 / 2}^{h}(\delta \mathbf{u})_{j+1 / 2}-\frac{1}{2}\left(\delta\left(\mathbf{u}^{2}\right)\right)_{j+1 / 2}-\dot{x}_{j-1 / 2}^{h}(\delta \mathbf{u})_{j-1 / 2}+\frac{1}{2} \delta\left(\mathbf{u}^{2}\right)_{j-1 / 2}\right]$

In section 4,we consider various temporal discretization of(3.3) and the stability of the resulting fully discrete schemes. The analysis will be carried out using the following mesh dependent norms. For the numerical solution, we use the $L_{2}$ norm.(noting the Homogeneous boundary conditions)

$$
\begin{aligned}
& \|\mathbf{u}\|_{n+\theta}=\left(\sum_{j=1}^{N-1}\left(\frac{h_{j+1}^{\theta}+h_{j}^{\theta}}{2}\right)\left(u_{j}\right)^{2}\right)^{1 / 2} \\
& \left\langle u, v>_{n+\theta}=\sum_{j=1}^{N-1}\left(\frac{h_{j+1}^{\theta}+h_{j}^{\theta}}{2}\right) u_{j} v_{j}\right.
\end{aligned}
$$

Approximations of the derivatives will be measured in the cell-based norm.

$$
\begin{gathered}
<u, v>\frac{}{(n+\theta)}=\sum_{j=1}^{N-1} h_{j}^{\theta} v_{j} w_{j} \\
\|v\|_{\overline{n+\theta}}=\left[\sum_{j=1}^{N}\left(h_{j}^{\theta}\right)\left(v_{j}\right)^{2}\right]^{1 / 2} \\
(\mu f)_{j}=\frac{f_{j+1}-f_{j-1}}{h_{j+1}+h_{j}}
\end{gathered}
$$

\section{Stability analysis of Modified Average Method using Moving Mesh}

For one dimensional non linear Burgers equation forward Euler as well as Backward Euler method are unstable. These methods are unstable due to the nonlinear term $\left(u^{2}\right)_{x}$. To avoid the nonlinearity, in Backward Euler method, we approximate $\left(u^{2}\right)_{x}$ by central difference at $t=t_{n}$. This gives Mixed Euler Method[23] which is superior than the existing methods.Therefore it is natural to extend this finite difference scheme for moving mesh.

\subsection{Modified Average method}

Discretizing (3.4) using Mixed Euler temporal discretization and assuming that $t_{n+1}-t_{n}=\Delta t$ yields the fully discrete scheme.

$$
\begin{aligned}
& \left(x_{\xi}^{h} u\right)_{j}^{n+1}=\left(x_{\xi}^{h} u\right)_{j}^{n}+ \\
& \frac{\Delta t}{\Delta \xi}\left[\left(k\left(D_{+}-D_{-}\right) \mathbf{u}\right)_{j}^{n+1}+\dot{x}_{j+1 / 2}^{h}(\delta \mathbf{u})_{j+1 / 2}^{n+1}-\frac{1}{2}\left(\delta\left(\mathbf{u}^{2}\right)\right)_{j+1 / 2}^{n}-\dot{x}_{j-1 / 2}^{h}(\delta \mathbf{u})_{j-1 / 2}^{n+1}+\frac{1}{2} \delta\left(\mathbf{u}^{2}\right)_{j-1 / 2}^{n}\right]
\end{aligned}
$$

Discretizing (3.4) using Forward Euler temporal discretization

$$
\begin{aligned}
& \left(x_{\xi}^{h} u\right)_{j}^{n+1}=\left(x_{\xi}^{h} u\right)_{j}^{n}+ \\
& \frac{\Delta t}{\Delta \xi}\left[\left(k\left(D_{+}-D_{-}\right) \mathbf{u}\right)_{j}^{n}+\dot{x}_{j+1 / 2}^{h}(\delta \mathbf{u})_{j+1 / 2}^{n}-\frac{1}{2}\left(\delta\left(\mathbf{u}^{2}\right)\right)_{j+1 / 2}^{n}-\dot{x}_{j-1 / 2}^{h}(\delta \mathbf{u})_{j-1 / 2}^{n}+\frac{1}{2} \delta\left(\mathbf{u}^{2}\right)_{j-1 / 2}^{n}\right]
\end{aligned}
$$

Now Modified Mixed Euler Method is

$$
\left(x_{\xi}^{h} u\right)_{j}^{n+1}=\left(x_{\xi}^{h} u\right)_{j}^{n}+\frac{\Delta t}{\Delta \xi}\left[\begin{array}{l}
\left(k\left(\frac{u_{j+1}^{n+1}-u_{j}^{n+1}}{h_{j+1}^{\theta}}\right)-k\left(\frac{u_{j}^{n+1}-u_{j-1}^{n+1}}{h_{j}^{\theta}}\right)\right) \\
+\dot{x}_{j+1 / 2}^{h}(\delta \mathbf{u})_{j+1 / 2}^{n+1}-\frac{1}{2}\left(\delta\left(\mathbf{u}^{2}\right)\right)_{j+1 / 2}^{n}-\dot{x}_{j-1 / 2}^{h}(\delta \mathbf{u})_{j-1 / 2}^{n+1}+\frac{1}{2} \delta\left(\mathbf{u}^{2}\right)_{j-1 / 2}^{n}
\end{array}\right]
$$

Where $h_{j}^{\theta}=\theta h_{j}^{n+1}+(1-\theta) h_{j}^{n}$ 

and Modified Forward Euler scheme is

$$
\left(x_{\xi}^{h} u\right)_{j}^{n+1}=\left(x_{\xi}^{h} u\right)_{j}^{n}+\frac{\Delta t}{\Delta \xi}\left[\begin{array}{l}
\left(k\left(\frac{u_{j+1}^{n}-u_{j}^{n}}{h_{j+1}^{\theta}}\right)-k\left(\frac{u_{j}^{n}-u_{j-1}^{n}}{h_{j}^{\theta}}\right)\right) \\
+\dot{x}_{j+1 / 2}^{h}(\delta \mathbf{u})_{j+1 / 2}^{n}-\frac{1}{2}\left(\delta\left(\mathbf{u}^{2}\right)\right)_{j+1 / 2}^{n}-\dot{x}_{j-1 / 2}^{h}(\delta \mathbf{u})_{j-1 / 2}^{n}+\frac{1}{2} \delta\left(\mathbf{u}^{2}\right)_{j-1 / 2}^{n}
\end{array}\right]
$$

Let us consider a weighted combination of the modified ME and FE schemes of the form

$$
\begin{aligned}
\left(x_{\xi}^{h} u\right)_{j}^{n+1}=\left(x_{\xi}^{h} u\right)_{j}^{n} & \theta(4.1)+(1-\theta)(4.2) \quad \text { which gives } \\
& +\frac{\Delta t}{\Delta \xi}\left\{k\left[\theta\left(\frac{u_{j+1}^{n+1}-u_{j}^{n+1}}{h_{j+1}^{\theta}}-\frac{u_{j}^{n+1}-u_{j-1}^{n+1}}{h_{j}^{\theta}}\right)+(1-\theta)\left(\frac{u_{j+1}^{n}-u_{j}^{n}}{h_{j+1}^{\theta}}-\frac{u_{j}^{n}-u_{j-1}^{n}}{h_{j}^{\theta}}\right)\right]\right. \\
& +\dot{x}_{j+1 / 2}^{h}\left(\theta(\delta u)_{j+1 / 2}^{n+1}+(1-\theta)(\delta u)_{j+1 / 2}^{n}\right)-\dot{x}_{j-1 / 2}^{h}\left(\theta(\delta u)_{j-1 / 2}^{n+1}-(1-\theta)(\delta u)_{j-1 / 2}^{n}\right) \\
& \left.-\frac{1}{2}\left(\delta\left(u^{2}\right)_{j+1 / 2}^{n}-\delta\left(u^{2}\right)_{j-1 / 2}^{n}\right)\right\}
\end{aligned}
$$

We call scheme (4.3) as Modified Average scheme with Moving mesh.

For this scheme we have the following stability result.

Theorem 4.1 The numerical solution of equation (2.1) obtained by equation (4.3) with $\theta=\max _{j} \frac{\sqrt{\left(x_{\xi}^{h}\right)_{j}^{n+1}}}{\sqrt{\left(x_{\xi}^{h}\right)_{j}^{n+1}}+\sqrt{\left(x_{\xi}^{h}\right)_{j}^{n}}}$ is bounded and the following a priori bound holds.

$\left\|\mathbf{u}^{n+1}\right\|_{n+1}^{2}=\left\|\mathbf{u}^{n}\right\|_{n}^{2}-2 k \Delta t\left\langle\theta D_{+} u^{n+1}+(1-\theta) D_{+} u^{n}, \theta D_{+} u^{n+1}+(1-\theta) D_{+} u^{n}\right\rangle_{\overline{n+\theta}}$

$-\Delta t\left\langle\mu\left(u^{2}\right)^{n}, \theta u^{n+1}+(1-\theta) u^{n}\right\rangle_{n+\theta}$

Proof:- Multiplying throught the Modified average Method (4.3) by $\theta u_{j}^{n+1}+(1-\theta) u_{j}^{n}$ we obtain

$$
\theta^{2}(\overline{M E}) u_{j}^{n+1}+\theta(1-\theta)(\overline{M E}) u_{j}^{n}+\theta(1-\theta)(\overline{F E}) u_{j}^{n+1}+(\theta)^{2} u_{j}^{n}(\overline{F E})
$$

Where $\overline{M E}$ and $\overline{F E}$ denote the Modified Mixed Euler and Modified Forward Euler schemes.

To evaluate

$$
\sum_{j=1}^{N-1}(4.5)
$$

We first evaluate

$$
\sum_{j=1}^{N-1}(\overline{M E}) u_{j}^{n}
$$

Multiply (4.1) by $u_{j}^{n}$ and sum over all interior nodes we obtain

$$
\sum_{j=1}^{N-1}\left(x_{\xi}^{h} u\right)_{j}^{n+1} u_{j}^{n}=\sum_{j=1}^{N-1}\left(x_{\xi}^{h} u\right)_{j}^{n} u_{j}^{n}+I+I I
$$

Using the identity $\mathrm{ab}=\frac{1}{2} a^{2}+\frac{1}{2} b^{2}-\frac{1}{2}(a-b)^{2}$

to $u_{j}^{n+1} u_{j}^{n}$ in 1.h.s gives 


$$
\frac{1}{2} \sum_{j=1}^{N-1}\left(x_{\xi}^{h}\right)_{j}^{n+1}\left(u_{j}^{n+1}\right)^{2}+\frac{1}{2} \sum_{j=1}^{N-1}\left(x_{\xi}^{h}\right)_{j}^{n+1}\left(u_{j}^{n}\right)^{2}-\frac{1}{2} \sum_{j=1}^{N-1}\left(x_{\xi}^{h}\right)_{j}^{n+1}\left(u_{j}^{n+1}-u_{j}^{n}\right)^{2}=\sum_{j=1}^{N-1}\left(x_{\xi}^{h}\right)_{j}^{n}\left(u_{j}^{n}\right)^{2}+I+I I
$$

Using (3.3) we get

$$
\begin{aligned}
\frac{1}{2} \sum_{j=1}^{N-1}\left(x_{\xi}^{h}\right)_{j}^{n+1}\left(u_{j}^{n+1}\right)^{2}+\frac{\Delta t}{2 \Delta \xi}\left(\dot{x}_{j+1 / 2}^{h}-\dot{x}_{j-1 / 2}^{h}\right)\left(u_{j}^{n}\right)^{2} \\
=\sum_{j=1}^{N-1}\left(x_{\xi}^{h}\right)_{j}^{n}\left(u_{j}^{n}\right)^{2}+\frac{1}{2} \sum_{j=1}^{N-1}\left(x_{\xi}^{h}\right)_{j}^{n+1}\left(u_{j}^{n+1}-u_{j}^{n}\right)^{2}+I+I I
\end{aligned}
$$

Where

And

$$
\begin{aligned}
\mathrm{I} & =k \frac{\Delta t}{\Delta \xi} \sum_{j=1}^{N-1}\left(\frac{u_{j+1}^{n+1}-u_{j}^{n+1}}{h_{j+1}^{\theta}}-\frac{u_{j}^{n+1}-u_{j-1}^{n+1}}{h_{j}^{\theta}}\right) u_{j}^{n} \\
& =-k \frac{\Delta t}{\Delta \xi}\left\langle D_{+} u^{n+1}, D_{+} u^{n}\right\rangle_{\overline{n+\theta}}
\end{aligned}
$$

$$
\begin{gathered}
I I=\frac{\Delta t}{\Delta \xi} \sum_{j=1}^{N-1}\left\{\dot{x}_{j+1 / 2}^{h}(\delta u)_{j+1 / 2}^{n+1}-\dot{x}_{j-1 / 2}^{h}(\delta u)_{j-1 / 2}^{n+1}-\frac{1}{2} \delta\left(u^{2}\right)_{j+1 / 2}^{n}+\frac{1}{2} \delta\left(u^{2}\right)_{j-1 / 2}^{n}\right\} u_{j}^{n} \\
=\frac{\Delta t}{2 \Delta \xi} \sum_{j=1}^{N-1}\left[\dot{x}_{j+1 / 2}^{h}\left(u_{j+1}^{n+1}+u_{j}^{n+1}\right)-\dot{x}_{j-1 / 2}^{h}\left(u_{j}^{n+1}+u_{j-1}^{n+1}\right)-\frac{1}{2}\left(\left(u^{2}\right)_{j+1}^{n}-\left(u^{2}\right)_{j-1}^{n}\right)\right] u_{j}^{n}
\end{gathered}
$$

To evaluate

$$
\sum_{j=1}^{N-1}(\overline{F E}) u_{j}^{n+1}
$$

Multiply (4.2) by $u_{j}^{n+1}$ and sum over all interior nodes we obtain

$$
\begin{gathered}
\sum_{j=1}^{N-1}\left(x_{\xi}^{h}\right)_{j}^{n+1}\left(u_{j}^{n+1}\right)^{2}=\sum_{j=1}^{N-1}\left(x_{\xi}^{h} u\right)_{j}^{n} u_{j}^{n+1}+I I I+I V \\
\therefore \text { using }(3.3) \text { we get } \\
\frac{1}{2} \sum_{j=1}^{N-1}\left(x_{\xi}^{h}\right)_{j}^{n+1}\left(u_{j}^{n+1}\right)^{2}=\frac{1}{2} \sum_{j=1}^{N-1}\left(x_{\xi}^{h}\right)_{j}^{n}\left(u_{j}^{n}\right)^{2}-\frac{1}{2} \sum_{j=1}^{N-1}\left(x_{\xi}^{h}\right)_{j}^{n}\left(u_{j}^{n}-u_{j}^{n+1}\right)^{2} \\
-\frac{\Delta t}{2 \Delta \xi} \sum_{j=1}^{N-1}\left(\dot{x}_{j+1 / 2}^{h}-\dot{x}_{j-1 / 2}^{h}\right)\left(u_{j}^{n+1}\right)^{2}+I I I+I V
\end{gathered}
$$

Where

And

$$
\begin{aligned}
\mathrm{III} & =k \frac{\Delta t}{\Delta \xi} \sum_{j=1}^{N-1}\left(\frac{u_{j+1}^{n}-u_{j}^{n}}{h_{j+1}^{\theta}}-\frac{u_{j}^{n}-u_{j-1}^{n}}{h_{j}^{\theta}}\right) u_{j}^{n+1} \\
& =-k \frac{\Delta t}{\Delta \xi}\left\langle D_{+} u^{n}, D_{+} u^{n+1}\right\rangle_{\overline{n+\theta}}
\end{aligned}
$$

$$
\begin{gathered}
I V=\frac{\Delta t}{\Delta \xi} \sum_{j=1}^{N-1}\left\{\dot{x}_{j+1 / 2}^{h}(\delta u)_{j+1 / 2}^{n}-\dot{x}_{j-1 / 2}^{h}(\delta u)_{j-1 / 2}^{n}-\frac{1}{2} \delta\left(u^{2}\right)_{j+1 / 2}^{n}+\frac{1}{2} \delta\left(u^{2}\right)_{j-1 / 2}^{n}\right\} u_{j}^{n+1} \\
=\frac{\Delta t}{2 \Delta \xi} \sum_{j=1}^{N-1}\left[\dot{x}_{j+1 / 2}^{h}\left(u_{j+1}^{n}+u_{j}^{n}\right)-\dot{x}_{j-1 / 2}^{h}\left(u_{j}^{n}+u_{j-1}^{n}\right)-\frac{1}{2}\left(\left(u^{2}\right)_{j+1}^{n}-\left(u^{2}\right)_{j-1}^{n}\right)\right] u_{j}^{n+1}
\end{gathered}
$$


To evaluate

$$
\sum_{j=1}^{N-1}(\overline{M E}) u_{j}^{n+1}
$$

Multiply (4.1) by $u_{j}^{n+1}$ and sum over all interior nodes we obtain

Using 4.6

$$
\sum_{j=1}^{N-1}\left(x_{\xi}^{h} u\right)_{j}^{n+1} u_{j}^{n+1}=\sum_{j=1}^{N-1}\left(x_{\xi}^{h} u\right)_{j}^{n} u_{j}^{n+1}+I+I I
$$

Using 3.3

$$
\sum_{j=1}^{N-1}\left(x_{\xi}^{h}\right)_{j}^{n+1}\left(u_{j}^{n+1}\right)^{2}=\frac{1}{2} \sum_{j=1}^{N-1}\left(x_{\xi}^{h}\right)_{j}^{n}\left[\left(u_{j}^{n+1}\right)^{2}+\left(u_{j}^{n}\right)^{2}-\left(u_{j}^{n+1}-u_{j}^{n}\right)^{2}\right]+V+V I
$$

$$
\begin{aligned}
\frac{1}{2} \sum_{j=1}^{N-1}\left(x_{\xi}^{h}\right)_{j}^{n+1}\left(u_{j}^{n+1}\right)^{2} & =\frac{1}{2} \sum_{j=1}^{N-1}\left[\left(x_{\xi}^{h}\right)_{j}^{n+1}-\frac{\Delta t}{\Delta \xi}\left(\dot{x}_{j+1 / 2}^{h}-\dot{x}_{j-1 / 2}^{h}\right)\right]\left(u_{j}^{n+1}\right)^{2} \\
+ & \frac{1}{2} \sum_{j=1}^{N-1}\left(x_{\xi}^{h}\right)_{j}^{n}\left(u_{j}^{n}\right)^{2}-\frac{1}{2} \sum_{j=1}^{N-1}\left(x_{\xi}^{h}\right)_{j}^{n}\left(u_{j}^{n+1}-u_{j}^{n}\right)^{2}+V+V I
\end{aligned}
$$

Where

And

$$
\begin{aligned}
\mathrm{V} & =k \frac{\Delta t}{\Delta \xi}\left\{\sum_{j=1}^{N-1} \frac{u_{j+1}^{n+1}-u_{j}^{n+1}}{h_{j+1}^{\theta}} u_{j}^{n+1}-\sum_{j=1}^{N-1} \frac{u_{j}^{n+1}-u_{j-1}^{n+1}}{h_{j}^{\theta}} u_{j}^{n+1}\right\} \\
& =-k \frac{\Delta t}{\Delta \xi}\left\langle D_{+} u^{n+1}, D_{+} u^{n+1}\right\rangle_{\overline{n+\theta}}
\end{aligned}
$$

$$
\begin{gathered}
V I=\frac{\Delta t}{2 \Delta \xi} \sum_{j=1}^{N-1}\left[\dot{x}_{j+1 / 2}^{h}\left(u_{j+1}^{n+1}+u_{j}^{n+1}\right)-\dot{x}_{j-1 / 2}^{h}\left(u_{j}^{n+1}+u_{j-1}^{n+1}\right)-\frac{1}{2}\left(\left(u^{2}\right)_{j+1}^{n}-\left(u^{2}\right)_{j-1}^{n}\right)\right] u_{j}^{n+1} \\
\frac{\Delta t}{2 \Delta \xi} \sum_{j=1}^{N-1}\left(\dot{x}_{j+1 / 2}^{h}-\dot{x}_{j-1 / 2}^{h}\right)\left(u_{j}^{n+1}\right)^{2}-\frac{\Delta t}{2 \Delta \xi}\left\langle\mu\left(u^{2}\right)^{n}, u^{n+1}\right\rangle_{n+\theta}
\end{gathered}
$$

To evaluate

$$
\sum_{j=1}^{N-1}(\overline{F E}) u_{j}^{n}
$$

Multiply (4.2) by $u_{j}^{n}$ and sum over all interior nodes we obtain

Using 4.6 and 3.3

$$
\sum_{j=1}^{N-1}\left(x_{\xi}^{h} u\right)_{j}^{n+1} u_{j}^{n}=\sum_{j=1}^{N-1}\left(x_{\xi}^{h} u\right)_{j}^{n} u_{j}^{n}+V I I+V I I I
$$

$$
\begin{gathered}
\frac{\mathbf{1}}{\mathbf{2}} \sum_{j=1}^{N-1}\left(x_{\xi}^{h}\right)_{j}^{n+1}\left(u_{j}^{n+1}\right)^{2}+\frac{1}{2} \sum_{j=1}^{N-1}\left[\left(x_{\xi}^{h}\right)_{j}^{n}+\frac{\Delta t}{\Delta \xi}\left(\dot{x}_{j+1 / 2}^{h}-\dot{x}_{j-1 / 2}^{h}\right)\right]\left(u_{j}^{n}\right)^{2}-\frac{1}{2} \sum_{j=1}^{N-1}\left(x_{\xi}^{h}\right)_{j}^{n+1}\left(u_{j}^{n+1}-u_{j}^{n}\right)^{2} \\
=\sum_{j=1}^{N-1}\left(x_{\xi}^{h} u\right)_{j}^{n} u_{j}^{n}+V I I+V I I I
\end{gathered}
$$

Where $V I I=k \frac{\Delta t}{\Delta \xi}\left\{\sum_{j=1}^{N-1} \frac{u_{j+1}^{n}-u_{j}^{n}}{h_{j+1}^{\theta}} u_{j}^{n}-\sum_{j=0}^{N-2} \frac{u_{j+1}^{n}-u_{j+1}^{n}}{h_{j+1}^{\theta}} u_{j+1}^{n}\right\}$ 
And

$$
=-k \frac{\Delta t}{\Delta \xi}\left\langle D_{+} u^{n}, D_{+} u^{n}\right\rangle_{\overline{n+\theta}}
$$

$$
\begin{gathered}
V I I I=\frac{\Delta t}{2 \Delta \xi} \sum_{j=1}^{N-1}\left[\dot{x}_{j+1 / 2}^{h}\left(u_{j}^{n}+u_{j+1}^{n}\right)-\dot{x}_{j-1 / 2}^{h}\left(u_{j}^{n}+u_{j-1}^{n}\right)-\frac{1}{2}\left(\left(u^{2}\right)_{j+1}^{n}-\left(u^{2}\right)_{j-1}^{n}\right)\right] u_{j}^{n} \\
=\frac{\Delta t}{2 \Delta \xi} \sum_{j=1}^{N-1}\left(\dot{x}_{j+1 / 2}^{h}-\dot{x}_{j-1 / 2}^{h}\right)\left(u_{j}^{n}\right)^{2}-\frac{\Delta t}{2 \Delta \xi}\left\langle\mu\left(u^{2}\right)^{n}, u^{n}\right\rangle_{n+\theta}
\end{gathered}
$$

Noting that

$\theta^{2}+2 \theta(1-\theta)+(1-\theta)^{2}=1$ and substituting (4.8),(4.9).(4.10) and (4.11) in (4.6) we get

Where

$$
\begin{aligned}
& \frac{1}{2}\left\|u^{n+1}\right\|_{n+1}^{2}=\frac{1}{2}\left\|u^{n}\right\|_{n}^{2}+\frac{(1-\theta)}{2}\left\|u^{n+1}-u^{n}\right\|_{n+1}^{2} \frac{-\theta}{2}\left\|u^{n+1}-u^{n}\right\|_{n}^{2}-(1-\theta)^{2} k \Delta t\left\|D_{+} u^{n}\right\|_{n+\theta}^{2} \\
& -(\theta)^{2} k \Delta t\left\|D_{+} u^{n+1}\right\|_{n+\theta}^{2}-\frac{\Delta t \theta^{2}}{4}\left[\left\|\mu\left(u^{2}\right)^{n}\right\|_{n+\theta}^{2}+\left\|u^{n+1}\right\|_{n+\theta}^{2}\right] \\
& \quad-\frac{\Delta t}{2}(1-\theta)^{2}\left\langle\mu\left(u^{2}\right)^{n}, u^{n}\right\rangle_{n+\theta}+I X+X+X I
\end{aligned}
$$

$$
\begin{gathered}
I X=\frac{-\theta(1-\theta)}{2} \Delta \xi \sum_{j=1}^{N-1}\left(u_{j}^{n+1}-u_{j}^{n}\right)^{2}\left[\left(x_{\xi}^{h}\right)_{j}^{n+1}-\left(x_{\xi}^{h}\right)_{j}^{n}\right] \\
\mathrm{X}=k \theta(1-\theta) \Delta t \sum_{j=1}^{N-1}\left(\frac{u_{j+1}^{n+1}-u_{j}^{n+1}}{h_{j+1}^{\theta}}-\frac{u_{j}^{n+1}-u_{j-1}^{n+1}}{h_{j}^{\theta}}\right) u_{j}^{n}+\left(\frac{u_{j+1}^{n}-u_{j}^{n}}{h_{j+1}^{\theta}}-\frac{u_{j}^{n}-u_{j-1}^{n}}{h_{j}^{\theta}}\right) u_{j}^{n+1} \\
=-2 k \theta(1-\theta) \Delta t \sum_{j=0}^{N-1}\left(D_{+} u^{n+1}\right)_{j}\left(D_{+} u^{n}\right)_{j} h_{j+1}^{\theta} \\
=-2 k \theta(1-\theta) \Delta t\left\langle D_{+} u^{n+1}, D_{+} u^{n+1}\right\rangle_{\overline{n+\theta}} \\
\mathrm{XI}=\frac{-\theta(1-\theta) \Delta t}{4} \sum_{j=1}^{N-1}\left(\left(u^{2}\right)_{j+1}^{n}-\left(u^{2}\right)_{j-1}^{n}\right) u_{j}^{n}+\left(\left(u^{2}\right)_{j+1}^{n}-\left(u^{2}\right)_{j-1}^{n}\right) u_{j}^{n+1} \\
=\frac{-\theta(1-\theta) \Delta t}{4}\left[\left\langle\mu\left(u^{2}\right)^{n}, u^{n+1}+u^{n}\right\rangle_{n+\theta}\right]
\end{gathered}
$$

We can see from (4.12) that the method will be stable if we can ensure that the term IX is a nonpositive. As this term arises from the mesh movement and has no physical meaning it would be ideal if we could choose $\theta$ so that it is zero.

We choose $\theta=\max _{j} \frac{\sqrt{\left(x_{\xi}^{h}\right)_{j}^{n+1}}}{\sqrt{\left(x_{\xi}^{h}\right)_{j}^{n+1}}+\sqrt{\left(x_{\xi}^{h}\right)_{j}^{n}}}$ as given in [22] so that IX becomes zero.

With this choice of $\theta$ and on simplifying X and XI and substituting in (4.12) we get (4.4)

Theorem 4.2 If

$\mathrm{A}=\left[\left\langle\mu\left(\varepsilon^{2}\right)^{n}, \theta \varepsilon^{n+1}+(1-\theta) \varepsilon^{n}\right\rangle_{n+\theta}+\left\langle\mu(u \varepsilon)^{n},(1-\theta)^{2} \varepsilon^{n}+\theta(2-\theta) \varepsilon^{n+1}\right\rangle_{n+\theta}\right] \geq 0$

then the scheme 4.3 is stable and

$$
\begin{gathered}
\left\|\varepsilon^{n+1}\right\|_{n+1}^{2}=\left\|\varepsilon^{n}\right\|_{n}^{2} \\
-2 k \Delta t\left\langle\theta D_{+} \varepsilon^{n+1}+(1-\theta) D_{+} \varepsilon^{n}, \theta D_{+} \varepsilon^{n+1}+(1-\theta) D_{+} \varepsilon^{n}\right\rangle_{\overline{n+\theta}}-A \Delta t
\end{gathered}
$$

Proof Let $\mathrm{U}$ be numerical solution and $\mathrm{u}$ be the exact solution of the difference scheme(4.3) then the error $\varepsilon=U-u$. Since $\mathrm{U}$ is the numerical solution of the scheme (4.3) 

$\left(x_{\xi}^{h} U\right)_{j}^{n+1}=\left(x_{\xi}^{h} U\right)$

$$
\begin{aligned}
& +\frac{\Delta t}{\Delta \xi}\left\{k\left[\theta\left(\frac{U_{j+1}^{n+1}-U_{j}^{n+1}}{h_{j+1}^{\theta}}-\frac{U_{j}^{n+1}-U_{j-1}^{n+1}}{h_{j}^{\theta}}\right)+(1-\theta)\left(\frac{U_{j+1}^{n}-U_{j}^{n}}{h_{j+1}^{\theta}}-\frac{U_{j}^{n}-U_{j-1}^{n}}{h_{j}^{\theta}}\right)\right]\right. \\
& +\dot{x}_{j+1 / 2}^{h}\left(\theta(\delta U)_{j+1 / 2}^{n+1}+(1-\theta)(\delta U)_{j+1 / 2}^{n}\right)-\dot{x}_{j-1 / 2}^{h}\left(\theta(\delta U)_{j-1 / 2}^{n+1}-(1-\theta)(\delta U)_{j-1 / 2}^{n}\right) \\
& \left.-\frac{1}{2}\left(\delta\left(U^{2}\right)_{j+1 / 2}^{n}-\delta\left(U^{2}\right)_{j-1 / 2}^{n}\right)\right\} \\
& \left(x_{\xi}^{h}(u+\varepsilon)\right)_{j}^{n+1}=\left(x_{\xi}^{h}(u+\varepsilon)\right)_{j}^{n} \\
& +\frac{\Delta t}{\Delta \xi}\left\{k \left[\theta\left(\frac{(u+\varepsilon)_{j+1}^{n+1}-(u+\varepsilon)_{j}^{n+1}}{h_{j+1}^{\theta}}-\frac{(u+\varepsilon)_{j}^{n+1}-(u+\varepsilon)_{j-1}^{n+1}}{h_{j}^{\theta}}\right)\right.\right. \\
& \left.+(1-\theta)\left(\frac{\left(x_{\xi}^{h}(u+\varepsilon)\right)_{j_{j+1}}^{n^{n}}-\left(x_{\xi}^{h}(u+\varepsilon)\right)_{j}^{n^{n}}}{h_{j+1}^{\theta}}-\frac{\left(x_{\xi}^{h}(u+\varepsilon)\right)_{j_{j}}^{n^{n}}-\left(x_{\xi}^{h}(u+\varepsilon)\right)_{j_{j-1}}^{n^{n}}}{h_{j}^{\theta}}\right)\right] \\
& +\dot{x}_{j+1 / 2}^{h}\left(\theta\left(\delta\left(x_{\xi}^{h}(u+\varepsilon)\right)_{j}^{n}\right)_{j+1 / 2}^{n+1}+(1-\theta)\left(\delta\left(x_{\xi}^{h}(u+\varepsilon)\right)_{j}^{n}\right)_{j+1 / 2}^{n}\right) \\
& -\dot{x}_{j-1 / 2}^{h}\left(\theta\left(\delta\left(x_{\xi}^{h}(u+\varepsilon)\right)_{j}^{n}\right)_{j-1 / 2}^{n+1}-(1-\theta)\left(\delta\left(x_{\xi}^{h}(u+\varepsilon)\right)_{j}^{n}\right)_{j-1 / 2}^{n}\right) \\
& \left.-\frac{1}{2}\left(\delta\left(\left(x_{\xi}^{h}(u+\varepsilon)\right)_{j}^{n^{2}}\right)_{j+1 / 2}^{n}-\delta\left(\left(x_{\xi}^{h}(u+\varepsilon)\right)_{j}^{n^{2}}\right)_{j-1 / 2}^{n}\right)\right\}
\end{aligned}
$$

The exact solution must satisfy difference equation 4.3 on subtracting equation (4.3) from (4.14) we get an error equation

$$
\begin{aligned}
\left(x_{\xi}^{h} \varepsilon\right)_{j}^{n+1}=\left(x_{\xi}^{h} \varepsilon\right)_{j}^{n} & \\
& +\frac{\Delta t}{\Delta \xi}\left\{k\left[\theta\left(\frac{\varepsilon_{j+1}^{n+1}-\varepsilon_{j}^{n+1}}{h_{j+1}^{\theta}}-\frac{\varepsilon_{j}^{n+1}-\varepsilon_{j-1}^{n+1}}{h_{j}^{\theta}}\right)+(1-\theta)\left(\frac{\varepsilon_{j+1}^{n}-\varepsilon_{j}^{n}}{h_{j+1}^{\theta}}-\frac{\varepsilon_{j}^{n}-\varepsilon_{j-1}^{n}}{h_{j}^{\theta}}\right)\right]\right. \\
& +\dot{x}_{j+1 / 2}^{h}\left(\theta(\delta \varepsilon)_{j+1 / 2}^{n+1}+(1-\theta)(\delta \varepsilon)_{j+1 / 2}^{n}\right)-\dot{x}_{j-1 / 2}^{h}\left(\theta(\delta \varepsilon)_{j-1 / 2}^{n+1}-(1-\theta)(\delta \varepsilon)_{j-1 / 2}^{n}\right) \\
& \left.-\frac{1}{2}\left(\delta\left(\varepsilon^{2}\right)_{j+1 / 2}^{n}-\delta\left(\varepsilon^{2}\right)_{j-1 / 2}^{n}\right)\right\}
\end{aligned}
$$

Multiplying 4.15 by $\theta \varepsilon_{j}^{n+1}+(1-\theta) \varepsilon_{j}^{n}$ we obtain

$$
\theta^{2}(\overline{E M E}) \varepsilon_{j}^{n+1}+\theta(1-\theta)(\overline{E M E}) \varepsilon_{j}^{n}+\theta(1-\theta)(\overline{E F E}) \varepsilon_{j}^{n+1}+(1-\theta)^{2} \varepsilon_{j}^{n}(\overline{E F E})
$$

Where $\overline{E M E}$ and $\overline{E F E}$ denote the error equation of Modified Mixed Euler and Modified forward Euler respectively.

To evaluate

We first evaluate

$$
\sum_{j=1}^{N-1}(4.16)
$$

$$
\sum_{j=1}^{N-1}(\overline{E M E}) \varepsilon_{j}^{n}
$$


Multiply errr equation of Modified Mixed Euler by $\varepsilon_{j}^{n}$ and sum over all interior nodes we obtain

$$
\sum_{j=1}^{N-1}\left(x_{\xi}^{h} \varepsilon\right)_{j}^{n+1} \varepsilon_{j}^{n}=\sum_{j=1}^{N-1}\left(x_{\xi}^{h} \varepsilon\right)_{j}^{n} \varepsilon_{j}^{n}+I+I I+I I I
$$

Using the identity 4.6 to $\varepsilon_{j}^{n+1} \varepsilon_{j}^{n}$ in 1.h.s gives

$$
\frac{1}{2} \sum_{j=1}^{N-1}\left(x_{\xi}^{h}\right)_{j}^{n+1}\left(\varepsilon_{j}^{n+1}\right)^{2}+\frac{1}{2} \sum_{j=1}^{N-1}\left(x_{\xi}^{h}\right)_{j}^{n+1}\left(\varepsilon_{j}^{n}\right)^{2}-\frac{1}{2} \sum_{j=1}^{N-1}\left(x_{\xi}^{h}\right)_{j}^{n+1}\left(\varepsilon_{j}^{n+1}-\varepsilon_{j}^{n}\right)^{2}=\sum_{j=1}^{N-1}\left(x_{\xi}^{h}\right)_{j}^{n}\left(\varepsilon_{j}^{n}\right)^{2}+I+I I+I I I
$$

Using 3.3

$$
\begin{gathered}
\frac{1}{2} \sum_{j=1}^{N-1}\left(x_{\xi}^{h}\right)_{j}^{n+1}\left(\varepsilon_{j}^{n+1}\right)^{2}+\frac{\Delta t}{2 \Delta \xi}\left(\dot{x}_{j+1 / 2}^{h}-\dot{x}_{j-1 / 2}^{h}\right)\left(\varepsilon_{j}^{n}\right)^{2} \\
=\sum_{j=1}^{N-1}\left(x_{\xi}^{h}\right)_{j}^{n}\left(\varepsilon_{j}^{n}\right)^{2}+\frac{1}{2} \sum_{j=1}^{N-1}\left(x_{\xi}^{h}\right)_{j}^{n+1}\left(\varepsilon_{j}^{n+1}-\varepsilon_{j}^{n}\right)^{2}+I+I I+I I I \\
\mathrm{I}=k \frac{\Delta t}{\Delta \xi} \sum_{j=1}^{N-1}\left(\frac{\varepsilon_{j+1}^{n+1}-\varepsilon_{j}^{n+1}}{h_{j+1}^{\theta}}-\frac{\varepsilon_{j}^{n+1}-\varepsilon_{j-1}^{n+1}}{h_{j}^{\theta}}\right) \varepsilon_{j}^{n} \\
=-k \frac{\Delta t}{\Delta \xi}\left\langle D_{+} \varepsilon^{n+1}, D_{+} \varepsilon^{n}\right\rangle_{n+\theta} \\
I I=\frac{\Delta t}{\Delta \xi} \sum_{j=1}^{N-1}\left\{\dot{x}_{j+1 / 2}^{h}(\delta \varepsilon)_{j+1 / 2}^{n+1}-\dot{x}_{j-1 / 2}^{h}(\delta \varepsilon)_{j-1}^{n+1} / 2-\frac{1}{2} \delta\left(\varepsilon^{2}\right)_{j+1 / 2}^{n}+\frac{1}{2} \delta\left(\varepsilon^{2}\right)_{j-1 / 2}^{n}\right\} \varepsilon_{j}^{n} \\
=\frac{\Delta t}{2 \Delta \xi} \sum_{j=1}^{N-1}\left[\dot{x}_{j+1 / 2}^{h}\left(\varepsilon_{j+1}^{n+1}+\varepsilon_{j}^{n+1}\right)-\dot{x}_{j-1 / 2}^{h}\left(\varepsilon_{j}^{n+1}+\varepsilon_{j-1}^{n+1}\right)-\frac{1}{2}\left(\left(\varepsilon^{2}\right)_{j+1}^{n}-\left(\varepsilon^{2}\right)_{j-1}^{n}\right)\right] \varepsilon_{j}^{n} \\
I I I=-\frac{\Delta t}{\Delta \xi} \sum_{j=1}^{N-1}\left(\delta(u \varepsilon)_{j+1 / 2}^{n}-\delta(u \varepsilon)_{j-1 / 2}^{n}\right) \varepsilon_{j}^{n} \\
=-\frac{\Delta t}{\Delta \xi}\left\langle\mu(u \varepsilon)^{n}, \varepsilon^{n}\right\rangle_{n+\theta}
\end{gathered}
$$

To evaluate

$$
\sum_{j=1}^{N-1}(\overline{E F E}) \varepsilon_{j}^{n+1}
$$

Multiply error equation of Modified Forward Euler by $\varepsilon_{j}^{n+1}$ and sum over all interior nodes we obtain

$$
\begin{gathered}
\sum_{j=1}^{N-1}\left(x_{\xi}^{h}\right)_{j}^{n+1}\left(\varepsilon_{j}^{n+1}\right)^{2}=\sum_{j=1}^{N-1}\left(x_{\xi}^{h} \varepsilon\right)_{j}^{n} \varepsilon_{j}^{n+1}+I V+V+V I \\
\frac{\mathbf{1}}{\mathbf{2}} \sum_{j=1}^{N-1}\left(x_{\xi}^{h}\right)_{j}^{n+1}\left(\varepsilon_{j}^{n+1}\right)^{2}=\frac{1}{2} \sum_{j=1}^{N-1}\left(x_{\xi}^{h}\right)_{j}^{n}\left(\varepsilon_{j}^{n}\right)^{2}-\frac{1}{2} \sum_{j=1}^{N-1}\left(x_{\xi}^{h}\right)_{j}^{n}\left(\varepsilon_{j}^{n}-\varepsilon_{j}^{n+1}\right)^{2} \\
-\frac{\Delta t}{2 \Delta \xi} \sum_{j=1}^{N-1}\left(\dot{x}_{j+1 / 2}^{h}-\dot{x}_{j-1 / 2}^{h}\right)\left(\varepsilon_{j}^{n+1}\right)^{2}+I V+V+V I
\end{gathered}
$$

Where

$$
\begin{aligned}
\mathrm{IV} & =k \frac{\Delta t}{\Delta \xi} \sum_{j=1}^{N-1}\left(\frac{\varepsilon_{j+1}^{n}-\varepsilon_{j}^{n}}{h_{j+1}^{\theta}}-\frac{\varepsilon_{j}^{n}-\varepsilon_{j-1}^{n}}{h_{j}^{\theta}}\right) \varepsilon_{j}^{n+1} \\
& =-k \frac{\Delta t}{\Delta \xi}\left\langle D_{+} \varepsilon^{n}, D_{+} \varepsilon^{n+1}\right\rangle_{\overline{n+\theta}}
\end{aligned}
$$


And

$$
\begin{gathered}
V=\frac{\Delta t}{\Delta \xi} \sum_{j=1}^{N-1}\left\{\dot{x}_{j+1 / 2}^{h}(\delta \varepsilon)_{j+1 / 2}^{n}-\dot{x}_{j-1 / 2}^{h}(\delta \varepsilon)_{j-1 / 2}^{n}-\frac{1}{2} \delta\left(\varepsilon^{2}\right)_{j+1 / 2}^{n}+\frac{1}{2} \delta\left(\varepsilon^{2}\right)_{j-1 / 2}^{n}\right\} \varepsilon_{j}^{n+1} \\
=\frac{\Delta t}{2 \Delta \xi} \sum_{j=1}^{N-1}\left[\dot{x}_{j+1 / 2}^{h}\left(\varepsilon_{j+1}^{n}+\varepsilon_{j}^{n}\right)-\dot{x}_{j-1 / 2}^{h}\left(\varepsilon_{j}^{n}+\varepsilon_{j-1}^{n}\right)-\frac{1}{2}\left(\left(\varepsilon^{2}\right)_{j+1}^{n}-\left(\varepsilon^{2}\right)_{j-1}^{n}\right)\right] \varepsilon_{j}^{n+1} \\
V I=-\frac{\Delta t}{\Delta \xi} \sum_{j=1}^{N-1}\left(\delta(u \varepsilon)_{j+1 / 2}^{n}-\delta(u \varepsilon)_{j-1 / 2}^{n}\right) \varepsilon_{j}^{n+1} \\
=-\frac{\Delta t}{\Delta \xi}\left\langle\mu(u \varepsilon)^{n}, \varepsilon^{n+1}\right\rangle_{n+\theta}
\end{gathered}
$$

To evaluate

$$
\sum_{j=1}^{N-1}(\overline{E M E}) \varepsilon_{j}^{n+1}
$$

Multiply error equation by $\varepsilon_{j}^{n+1}$ and sum over all interior nodes we obtain

$$
\sum_{j=1}^{N-1}\left(x_{\xi}^{h} \varepsilon\right)_{j}^{n+1} \varepsilon_{j}^{n+1}=\sum_{j=1}^{N-1}\left(x_{\xi}^{h} \varepsilon\right)_{j}^{n} \varepsilon_{j}^{n+1}+V I I+V I I I+I X
$$

Where

And

$$
\begin{aligned}
V I I & =k \frac{\Delta t}{\Delta \xi}\left\{\sum_{j=1}^{N-1} \frac{\varepsilon_{j+1}^{n+1}-\varepsilon_{j}^{n+1}}{h_{j+1}^{\theta}} \varepsilon_{j}^{n+1}-\sum_{j=1}^{N-1} \frac{\varepsilon_{j}^{n+1}-\varepsilon_{j-1}^{n+1}}{h_{j}^{\theta}} \varepsilon_{j}^{n+1}\right\} \\
& =-k \frac{\Delta t}{\Delta \xi}\left\langle D_{+} \varepsilon^{n+1}, D_{+} \varepsilon^{n+1}\right\rangle_{\overline{n+\theta}}
\end{aligned}
$$

$$
\begin{gathered}
V I I I=\frac{\Delta t}{2 \Delta \xi} \sum_{j=1}^{N-1}\left[\dot{x}_{j+1 / 2}^{h}\left(\varepsilon_{j+1}^{n+1}+\varepsilon_{j}^{n+1}\right)-\dot{x}_{j-1 / 2}^{h}\left(\varepsilon_{j}^{n+1}+\varepsilon_{j-1}^{n+1}\right)-\frac{1}{2}\left(\left(\varepsilon^{2}\right)_{j+1}^{n}-\left(\varepsilon^{2}\right)_{j-1}^{n}\right)\right] \varepsilon_{j}^{n+1} \\
=\frac{\Delta t}{2 \Delta \xi} \sum_{j=1}^{N-1}\left(\dot{x}_{j+1 / 2}^{h}-\dot{x}_{j-1 / 2}^{h}\right)\left(\varepsilon_{j}^{n+1}\right)^{2}-\frac{\Delta t}{2 \Delta \xi}\left\langle\mu\left(\varepsilon^{2}\right)^{n}, \varepsilon^{n+1}\right\rangle_{n+\theta}
\end{gathered}
$$

To evaluate

$$
\begin{aligned}
I X & =-\frac{\Delta t}{\Delta \xi} \sum_{j=1}^{N-1}\left(\delta(u \varepsilon)_{j+1 / 2}^{n}-\delta(u \varepsilon)_{j-1 / 2}^{n}\right) \varepsilon_{j}^{n+1} \\
& =-\frac{\Delta t}{\Delta \xi}\left\langle\mu(u \varepsilon)^{n}, \varepsilon^{n+1}\right\rangle_{n+\theta}
\end{aligned}
$$

$$
\sum_{j=1}^{N-1}(\overline{E F E}) \varepsilon_{j}^{n}
$$

Multiply error equation by $\varepsilon_{j}^{n}$ and sum over all interior nodes we obtain

$$
\sum_{j=1}^{N-1}\left(x_{\xi}^{h} \varepsilon\right)_{j}^{n+1} \varepsilon_{j}^{n}=\sum_{j=1}^{N-1}\left(x_{\xi}^{h} \varepsilon\right)_{j}^{n} \varepsilon_{j}^{n}+X+X I+X I I
$$

where

$$
\mathrm{X}=k \frac{\Delta t}{\Delta \xi}\left\{\sum_{j=1}^{N-1} \frac{\varepsilon_{j+1}^{n}-\varepsilon_{j}^{n}}{h_{j+1}^{\theta}} \varepsilon_{j}^{n}-\sum_{j=0}^{N-2} \frac{\varepsilon_{j+1}^{n}-\varepsilon_{j+1}^{n}}{h_{j+1}^{\theta}} \varepsilon_{j+1}^{n}\right\}
$$


And

$$
=-k \frac{\Delta t}{\Delta \xi}\left\langle D_{+} \varepsilon^{n}, D_{+} \varepsilon^{n}\right\rangle_{\overline{n+\theta}}
$$

$$
\begin{gathered}
X I=\frac{\Delta t}{2 \Delta \xi} \sum_{j=1}^{N-1}\left[\dot{x}_{j+1 / 2}^{h}\left(\varepsilon_{j}^{n}+\varepsilon_{j+1}^{n}\right)-\dot{x}_{j-1 / 2}^{h}\left(\varepsilon_{j}^{n}+\varepsilon_{j-1}^{n}\right)-\frac{1}{2}\left(\left(\varepsilon^{2}\right)_{j+1}^{n}-\left(\varepsilon^{2}\right)_{j-1}^{n}\right)\right] \varepsilon_{j}^{n} \\
=\frac{\Delta t}{2 \Delta \xi} \sum_{j=1}^{N-1}\left(\dot{x}_{j+1 / 2}^{h}-\dot{x}_{j-1 / 2}^{h}\right)\left(\varepsilon_{j}^{n}\right)^{2}-\frac{\Delta t}{2 \Delta \xi}\left\langle\mu\left(\varepsilon^{2}\right)^{n}, \varepsilon^{n}\right\rangle_{n+\theta} \\
X I I=-\frac{\Delta t}{\Delta \xi} \sum_{j=1}^{N-1}\left(\delta(u \varepsilon)_{j+1 / 2}^{n}-\delta(u \varepsilon)_{j-1 / 2}^{n}\right) \varepsilon_{j}^{n} \\
=-\frac{\Delta t}{\Delta \xi}\left\langle\mu(u \varepsilon)^{n}, \varepsilon^{n}\right\rangle_{n+\theta}
\end{gathered}
$$

Proceeding same way as in theorem 4,1 and substituting (4.18),(4.19),(4.20) and (4.21) in (4.17) we get 4.13

\section{Numerical Experiments and analysis}

Numerical solution of 1-dimensional nonlinear Burgers equation are obtained by Modified Average Method with moving mesh method by the difference formula (4.3). The solutions are obtained for $\mathrm{k}=1$ and $\mathrm{k}=0.5$ for $\mathrm{t}=0.1,0.15,0.2$ with $\Delta t=0.001 \& \Delta \xi=0.01$ for different domains. The graphs of solution in these domains are given for the analysis.

Example1:-To test the analysis, we consider the solution of (2.1) in the domain $x_{l}(t)=1-e^{t / 2}$ and $x_{r}(t)=e^{t / 2}$ with Homogeneous Dirichlets boundary conditions. We use a set of mesh points which are uniformly spaced between $x_{l}(t) \& x_{r}(t)$. The initial condition is taken to be $u(x, 0)=u_{0}(x)=\sin \pi x$ and we choose $\mathrm{k}=1$ and $\mathrm{k}=0.5$ with $\Delta t=0.001$ and $\Delta \xi=0.01$.Figure 1 and 2 shows the solution of (2.1) for different values of $\mathrm{t}$
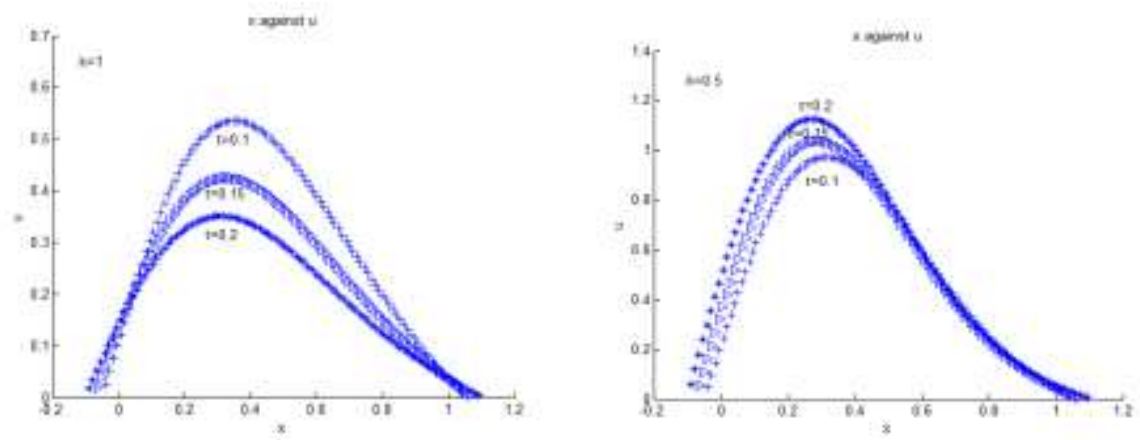

Example 2 Consider the one dimensional non linear Burgers equaion (2.1) in the domain $x_{l}(t)=0$ and $x_{r}(t)=e^{2 t}$ with Homogeneous Dirichlets boundary conditions and initial condition $u_{0}(x)=\frac{x_{r}-x}{x_{r}-x_{l}}$. we choose $\Delta t=0.001$ and $\Delta \xi=0.01$. The numerical solution of above equation with given initial and boundary conditions are shown in figure 1 and figure 2 for $\mathrm{k}=1$ and $\mathrm{k}=0.5$ respectively for different values of $\mathrm{t}$.
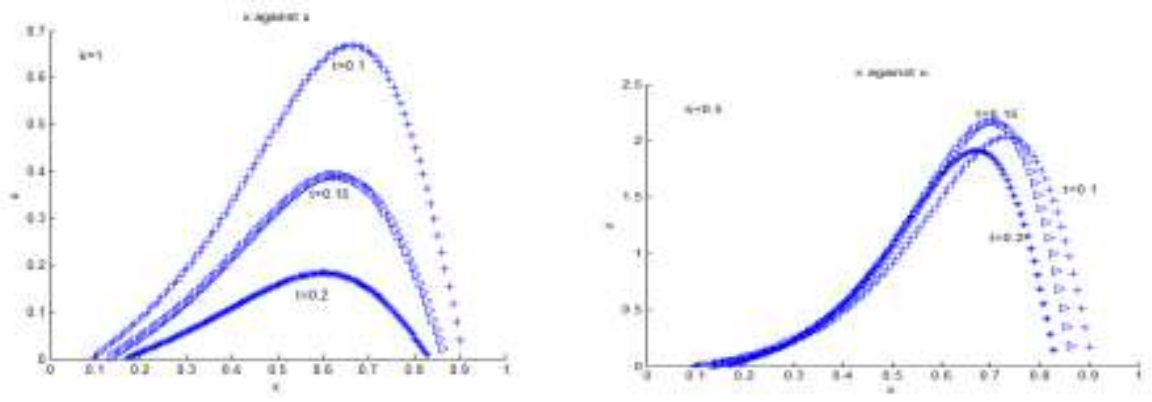


\section{Conclusion}

A Modified Average method with Moving Mesh is constructed for 1-D nonlinear Burgers equation with Homogeneous Dirichlets boundary conditions. For this method discretization of the terms containing linear differentials are approximated by backward difference where as the nonlinear terms by central difference in state variable. The solutions are proved to be bounded and the method is made stable in $L_{2}$ norm. The boundedness of error is achieved.Numerical solutions are obtained for different domains.

\section{References:-}

[1] HUANG . W . \& RUSSELL.R.D(2001), "Adaptive mesh movement- the MMPDE approach \& its applications". J. comput. Appl .math, 128,383-398.

[2] MADZVAMUSE,A.,MAINI,P.K.,WATHEN,A.J(2005), “A moving grid finite element method for the simulation of pattern generation by turning model on growing domains"J.Sci.Comput.,24,247-262.

[3] BAINE S , M . J . (2001), "Moving finite element, least squares ,\& finite volume approximations of steady \& time dependent PDE 's in multidimensions . J. comput .App . math,128,363-381.

[4] H.Beteman. "Some recent researches on the motion of fluids",Mon.Weather Rev.43(1915)163-170.

[5] J. M. Burger, "A Mathematical model illustrating the theory of turbulence” Adv.Appl.Mech 1(1948)171-199.

[6] E.Hopf. "The partial differential equation $u_{t}+u u_{x}=\mu u_{x x}$,, Commun, pure Appl.Math3 (1950)301-330.

[7] J.D.Cole, "On a quasi linear parabolic equations occurring in aerodynamics",Quart.Appl.Math9(1951)225-236.

[8] N.Su.Australia "Burgers equation for water dynamics in solids on Eroding Hillslopes proceeding 4(43) Applied simulation \& modeling 2004.

[9] Edris Dag Ali sahin, "Numerical solution of Burgers Equation over geometrically graded mesh" year 2007 volume 36 issue 510,721-735

[10] Ronald E.mickens, “ Exact solution to different equations models of Burgers equation”.Numerical methods for partial differential equation volume 2 issue page123-129-2005 A wiley company.

[11] Alireza,Hashemian,Hussein m shodja, "A meshless approach for solution of Burgers equation", Journal of computational and applied mathematics volume 220 issue 1-2 (October2008) 226-239.

[12] Sachin S.Wani,Sarita Thakar, "Stability Analysis of Mixed Euler method for one dimensional nonlinear Burgers equation", Bulletin of Kerala Mathematical Association December 2009 volume 5,no.2,pp19-31.

[13] FERREIRAJ.A(1997), “on the convergence of nonrectangular grids"Appl.Numer.Math.85,333-344

[14] THOMAS P.D. \& LOMBARD C.K(1979) "Geometric conservation law \& its application to flow computations on moving grids".AIAAJ,17,1030-1037.

[15] R.M.Furzeland.J.G.Verwew \& P.A.Zegeling. "A numerical study of three moving grid methods for 1 -d partial differential equation which are based on the methods of lines"J.Comp.Phy 89(1990)349-399

[16] E.A.Dorfi \& D.C.Drury "Simple adaptive grids for 1-d initial value problem” J.comp.phy 69(1987)175-195.

[17] Skell \& Berzins "A method for the spatial discretization of parabolic differential equation in one space variable"SIAM J.Sci,Statist,comp-11(1990)1-32.

[18] W.Z.Huang, "Practical aspects of formulation \& solution of moving mesh PDE's",J.comput.ph,95(1991)254-302.

[19] C.Budd Wz -Huang \& R.D.Russell, "Moving mesh methods for problems with blow up",SIAM J.Sci.comput-17(1996)305-327.

[20] Dupont,T.F \&LIU.Y.(2002) "Symmetric error estimstes for moving mesh Galerkin methods for advection diffusion equations",SIAM,J.Numer.Anal.40,914-927.

[21] LIU.Y.BANK,R.E.Dupont,T.F.Garcia,S \& Santos R.F(2003), "Symmetric error estimates for moving mesh mixed methods for advection diffusion equations",SIAM,J.Numer.Anal.40,2270-2291.

[22] Zhijum Tan,Zhengru,Zhang,Yunging,Huan Tao Tang "Moving mesh methods with locally varying time steps", J. Comp. Phys. 200 (2004), pp. 347-367.

[23] J.A.Mackenzie and W.R.Mekwi, "An analysis of stability \& convergence of a finite difference discretization of a model parabolic PDE in 1-D using a moving mesh". IMA journal of Numerical analysis (2007)27,507-528. 